\title{
Los problemas auditivos aumentan el riesgo de deterioro cognitivo en adultos mayores chilenos
}

\author{
Hearing impairments increase the risk of cognitive impairment \\ in older Chilean adults
}

\author{
Fanny Petermann R ${ }^{1}$, Claudia Troncoso-Pantoja ${ }^{2}$, María Adela Martínez $\mathrm{S}^{3}$, Ana María Leiva $\mathbf{0}^{4}$, \\ Natalia Ulloa $\mathbf{M}^{5}$ y Carlos Celis-Morales ${ }^{6}$.
}

\begin{abstract}
RESUMEN
Introducción: Estudios previos han asociado la pérdida auditiva con un acelerado deterioro cognitivo durante el envejecimiento; no obstante, esta asociación no ha sido estudiada en adultos mayores chilenos.

Objetivo: Investigar la asociación entre la discapacidad auditiva y la sospecha de deterioro cognitivo a través del cuestionario Mini Mental State Examination (MMSE, versión abreviada) en esta población.

Material y método: Se incluyeron 1.384 adultos mayores de 60 años de la encuesta nacional de salud 2009-2010. Un puntaje <13 puntos en el MMSE se consideró sospecha de deterioro cognitivo. La discapacidad auditiva se determinó a través de un cuestionario de tamizaje autorreportado de tres preguntas. La asociación entre estas dos variables se investigó mediante análisis de regresión logística.

Resultados: Se identificó una asociación significativa entre el MMSE alterado y cada una de las discapacidades auditivas estudiadas. A su vez, existió una tendencia a aumentar en 59\% la probabilidad de desarrollar deterioro cognitivo en la medida que aumentaron las discapacidades auditivas (OR: 1,59 [95\% IC: 1,38 a 1,82], $p<0,0001$ ).

Conclusión: La disminución de la percepción auditiva es un factor de riesgo para el desarrollo de deterioro cognitivo y posteriormente demencia. La creación de políticas públicas, orientadas al tamizaje temprano en población de riesgo, podría ser una solución efectiva para prevenir las consecuencias asociadas con esta condición.
\end{abstract}

Palabras clave: Envejecimiento, pruebas de estado mental y demencia, pérdida auditiva, deterioro cognitivo.

1 Nutrición Humana. BHF Glasgow Cardiovascular Research Centre, Institute of Cardiovascular and Medical Science, University of Glasgow, Glasgow, United Kingdom.

2 CIEDE-UCSC, Facultad de Medicina, Universidad Católica de la Santísima Concepción, Concepción, Chile.

3 Nutrición y Dietética. Instituto de Farmacia, Facultad de Ciencias, Universidad Austral de Chile, Valdivia, Chile.

4 Instituto de Anatomía, Histología y Patología, Facultad de Medicina, Universidad Austral de Chile, Valdivia, Chile.

5 Centro de Vida Saludable, Universidad de Concepción, Concepción, Chile.

6 BHF Glasgow Cardiovascular Research Centre, Institute of Cardiovascular and Medical Science, University of Glasgow, Glasgow, United Kingdom y Centro de Investigación en Fisiología del Ejercicio - CIFE, Universidad Mayor. Santiago, Chile.

*Los autores declaran no tener conflictos de interés.

Recibido el 19 de junio, 2018. Aceptado el 14 de agosto, 2018. 


\section{ABSTRACT}

Introduction: Hearing loss has been associated with an accelerated cognitive impairment during ageing. However, this association has not been investigated in older Chilean adults.

Aim: To investigate the association between hearing impairment and cognitive impairment, using the Mini-Mental State Examination (MMSE, abridged version), in the Chilean population.

Material and methods: 1,384 older adults aged $\geq 60$ years, from 2009-2010 Chilean national health survey, were included. $<13$ points in the MMSE were considered suspicion of cognitive impairment. Hearing impairment was determined through a questionnaire including 3 domains. The association between cognitive and hearing loss was investigated using logistic regression.

Results: An association between MMSE and each hearing disabilities studied was identified. As hearing impairments increased, the odd for cognitive impairment incremented by 59\% (OR: 1.59 [95\% IC: 1.38 a 1.82], $p<0.0001$ ).

Conclusion: Hearing loss is a risk factor to develop cognitive impairment, which could translate into a high risk of dementia. Public politics implementation, focused in an early screening, could be an effective approach to prevent the complications associated with this hearing loss in older adults.

Key words: Ageing, mental status and dementia tests, hearing impairment, cognitive dysfunction.

\section{INTRODUCCIÓN}

La discapacidad auditiva y cognitiva son condiciones altamente prevalentes entre los adultos mayores $^{1}$, siendo motivos de preocupación global y económica. Se estima que una tercera parte de las personas mayores de 65 años padecen pérdida de audición discapacitante, es decir, presentan una pérdida de audición superior 40 decibeles en el oído con mejor audición ${ }^{2}$; mientras que entre $5 \%$ a $8 \%$ de los adultos mayores de 60 años padecen alguna forma de demencia asociada a deterioro cognitivo ${ }^{3}$. La discapacidad auditiva, en cualquiera de sus dominios, afecta negativamente la comunicación, la salud, la calidad de vida, la confianza y la autoimagen de las personas que la padecen, siendo asociada con sentimientos de frustración, soledad, depresión, ansiedad, paranoia y confusión emocional ${ }^{4,5}$. Adicionalmente, numerosos estudios han asociados la pérdida auditiva con un acelerado deterioro cognitivo durante el envejecimiento $0^{6-9}, \mathrm{y}$, a su vez, el deterioro cognitivo se ha asociado con una mayor disminución de la sensibilidad auditiva ${ }^{9}$, existiendo una relación lineal entre estas dos condiciones ${ }^{6}$.
Uno de los instrumentos más utilizados para determinar sospecha de deterioro cognitivo en adultos mayores es el Mini-Mental test (Mini Mental State Examination, MMSE) ${ }^{10}$. El MMSE es una prueba breve de función cognitiva básica, creado por Folstein en el año 1975'11. En el año 1999, este test fue modificado en Chile ${ }^{12}$, lo que permitió obtener una versión abreviada del instrumento. Tras ser validado ${ }^{13}$, el MMSE abreviado se ha convertido en el instrumento gold standard para determinar sospecha de deterioro cognitivo en los adultos mayores del país ${ }^{14}$.

\section{OBJETIVO}

Considerando que el MMSE abreviado es un instrumento ampliamente utilizado en Chile, y que el $32,7 \%$ de la población adulta declara presentar al menos un problema de audición ${ }^{15}$, el objetivo de este estudio fue investigar la asociación entre la discapacidad auditiva y la sospecha de deterioro cognitivo a través del MMSE abreviado en adultos mayores chilenos. 


\section{MATERIAL Y MÉTODO}

\section{Diseño del estudio}

La muestra seleccionada comprende a todos los participantes mayores de 60 años de la Encuesta Nacional de Salud de Chile (ENS 2009-2010) que poseían información en relación al test MMSE y problemas de audición ${ }^{15}$. La ENS 2009-2010 correspondió a un estudio de prevalencia realizado en hogares en una muestra nacional, probabilística, estratificada y multietápica de 5.412 personas mayores de 15 años con representatividad nacional, regional, y área urbana/rural. En este estudio fueron incluidos 1.384 participantes (61\% mujeres). El protocolo de estudio fue aprobado por el Comité de Ética de la Escuela de Medicina de la Pontificia Universidad Católica de Chile. Todos los participantes firmaron un consentimiento informado ${ }^{15}$.

\section{Discapacidad auditiva}

La percepción y presencia de disminución de la agudeza auditiva se determinó a través de un cuestionario de tamizaje autorreportado de tres preguntas: (1) consideración de que escucha en forma normal por los dos oídos (A1), (2) capacidad de seguir un programa de televisión a un volumen aceptable (A2) y (3) capacidad de seguir una conversación de tres o más personas $(A 3)^{15}$.

\section{Test MMSE}

El cuestionario MMSE, versión abreviada, fue utilizado para identificar alteraciones cognitivas en los participantes. La versión abreviada consta de 6 preguntas, con un puntaje máximo de 19 puntos. Un puntaje menor a 13 se consideró sospecha de deterioro cognitivo ${ }^{15}$.

\section{Variables sociodemográficas, metabólicas, antropométricas y de estilo de vida}

Las variables sociodemográficas (edad, sexo, nivel educacional, ingreso económico [bajo $<\$ 250.000$; medio $\$ 250.000$ a 650.000 ; alto $>\$ 650.000$ ], zona geográfica [rural 0 urbana acorde a la clasificación otorgada por la ENS 2009-2010]) y datos asociados con estilo de vida, como el tabaquismo, horas de sueño, autorreporte de salud y bienestar, consumo de alcohol, frutas y verduras se obtuvieron mediante la aplicación de cuestionarios validados en la ENS 2009-2010'5.

Para la detección de diabetes mellitus tipo 2, se consideró una glicemia en ayuno $\geq 126 \mathrm{mg} / \mathrm{dl}$ 0 el autorreporte de diagnóstico médico de ésta ${ }^{16}$. Se consideró síndrome metabólico a la presencia de 30 más de los 5 criterios definidos en ATP III Update $2004^{17}$. La hipertensión arterial fue definida a través de tres mediciones $\geq 140 / 90 \mathrm{mmHg} 0$ autorreporte de tratamiento farmacológico para esta patología. El estado nutricional fue clasificado en base a los puntos de corte de valoración específica para el adulto mayor del índice de masa corporal (IMC: $\mathrm{kg} / \mathrm{m}^{2}$ ): bajo peso: $<22,9 \mathrm{~kg} / \mathrm{m}^{2}$; normo peso: $23,0-27,9 \mathrm{~kg} / \mathrm{m}^{2}$; sobrepeso: $28,0-31,9 \mathrm{~kg} /$ $\mathrm{m}^{2}$ y obesidad: $\geq 32,0 \mathrm{~kg} / \mathrm{m}^{2}{ }^{18}$, mientras que la obesidad central fue definida como un perímetro de cintura $\geq 83 \mathrm{~cm}$ para mujeres $\mathrm{y} \geq 88 \mathrm{~cm}$ para hombres, según los puntos de corte sugeridos por la ENS 2009-2010 en población chilena ${ }^{15}$. Los niveles de actividad física (AF), el tiempo destinado a las actividades de transporte activo, y las actividades de intensidad moderada o vigorosa, fueron determinados con el cuestionario Global Physical Activity Questionnaire ${ }^{19}$. La AF total es presentada como la suma del tiempo reportado en actividades de transporte, de intensidad moderada y vigorosa tanto en el trabajo como en el tiempo libre. Se consideró como punto de corte para inactividad física un gasto energético <600 METs/minutos/ semana. Los niveles de sedentarismo fueron determinados mediante el mismo cuestionario a partir del autorreporte de tiempo destinado a actividades que involucren estar sentado o reclinado durante el tiempo libre o de trabajo (ej.: tiempo sentado frente al computador o televisión, viajando en bus 0 auto, entre otras).

\section{Análisis estadístico}

Los datos de caracterización de la población estudiada son presentados como promedio y desviación estándar para variables continuas, y como porcentaje para variables categóricas. La asociación entre MMSE alterado (sospecha de deterioro cognitivo) y problemas auditivos (A1, A2 
y A3) fue investigada mediante análisis de regresión logística. Todos los análisis fueron ajustados por variables de confusión mediante la utilización de cinco modelos estadísticos: Modelo 0 - ajustado por sexo; Modelo 1 - ajustado por Modelo 0, pero también por nivel educacional y edad; Modelo 2 - ajustado por el Modelo 1, pero también por tabaquismo; Modelo 3 - ajustado por el Modelo 2, pero también por IMC; Modelo 4 - ajustado por el Modelo 2, pero también por perímetro de cintura. Los datos para estos análisis son presentados como Odds ratio (OR) y sus respectivos intervalos de confianza del $95 \%$ ( $95 \%$ IC). Para todos los análisis se utilizó el módulo de análisis de muestras complejas del programa STATA SE v14 y todos Ios resultados fueron estimados utilizando muestras expandidas según la ENS 2009-2010. El nivel de significancia fue definido como $p<0,05$.

\section{RESULTADOS}

Las características de la población estudiada según MMSE (normal versus alterado) se identifican en la Tabla 1.

En general, adultos mayores con MMSE alterado presentan una mayor edad, un menor nivel de ingresos y un nivel educativo más bajo en comparación a aquellos con MMSE normal. Poseen un menor peso corporal por lo que un mayor porcentaje de individuos presentan un estado nutricional de bajo peso. En relación con el estilo de vida, dedican un mayor tiempo diario en actividades de tipo sedentarias, realizan una menor cantidad de $\mathrm{AF}$ de transporte, moderada o vigorosa, duermen más horas diarias y poseen un peor autorreporte de salud y bienestar. Similarmente, un mayor porcentaje de adultos mayores con MMSE alterado presenta hipertensión arterial y discapacitades auditivas (A1, A2 y A3) en comparación con sus pares sin alteración. La asociación entre MMSE alterado (sospecha de deterioro cognitivo) y distintos tipos de discapacidad auditiva se observa en la Tabla 2.

Tal como se observa, existió una asociación significativa entre el MMSE alterado y cada una de las discapacidades auditivas estudiadas (A1, A2 y A3). En este contexto, existió una tendencia a incrementar en $59 \%$ la probabilidad de desarrollar deterioro cognitivo en la medida que aumentaron las discapacidades auditivas (OR: 1,59 [95\% IC: 1,38 a 1,82], $p<0,0001$ ) presentado $292 \%$ de mayor riesgo aquellos adultos mayores que poseían las 3 discapacidades estudiadas en comparación con sus pares sin problemas auditivos (OR: 3,91 [95\% IC: 2,53 a 6,06], p <0,0001) (Tabla 3). A pesar de que tras ajustar por las variables de confusión existió una disminución en la asociación de estas variables, ésta se mantuvo significativa incluso en los modelos más ajustados (Modelo 3 y Modelo 4). No se identificó una asociación significativa entre MMSE y la presencia de un solo problema auditivo en ninguno de los modelos.

\section{DISCUSIÓN}

La pérdida de la audición, relacionada con la edad, es concebida por muchos adultos mayores como un evento desafortunado, pero que forma parte del proceso de envejecimiento. No obstante, el principal problema al que se enfrentan estos adultos es la mayor probabilidad de padecer desórdenes del comportamiento, alteraciones del humor y psíquicas que se asocian principalmente al déficit cognitivo asociado a la discapacidad auditiva ${ }^{20,21}$. En este estudio, y tal como ha sido identificado previamente ${ }^{6-9}$, los adultos mayores que presentaron problemas auditivos evidenciaron un mayor riesgo de desarrollar deterioro cognitivo $y$, en consecuencia, una mayor probabilidad de desarrollar demencia. Este riesgo fue tres veces mayor en aquellos adultos mayores que exhibían los tres problemas de audición estudiados (A1, A2 y A3). Esta asociación se mantuvo incluso en los modelos más ajustados (OR: 2,63 y OR: 2,38, para Modelo 3 y 4, respectivamente). Por otro lado, y a pesar de que en este estudio no se identificó presencia de hipoacusia (pérdida parcial de la capacidad auditiva), lo que podría disminuir el poder estadístico de los resultados, los datos obtenidos a través de la alteración de la percepción auditiva fueron suficientes para demostrar una asociación entre esta variable y la probabilidad de desarrollar problemas cognitivos.

A pesar de que diversos estudios en Chile han investigado independientemente el deterioro cognitivo y la discapacidad auditiva en adultos ma- 
Tabla 1. Características de la población según Mini-Mental (MMSE). Datos son presentados como promedio y desviación estándar para variables continuas, y como porcentaje para variables categóricas

\begin{tabular}{|c|c|c|c|}
\hline & & $\begin{array}{l}\text { MMSE } \\
\text { normal }\end{array}$ & $\begin{array}{r}\text { MMSE } \\
\text { alterado }\end{array}$ \\
\hline $\begin{array}{l}\text { Sociodemográficas } \\
n \\
\text { Mujeres (\%) } \\
\text { Edad (años) }\end{array}$ & & $\begin{array}{r}1.215 \\
61,2 \\
69,9(7,4)\end{array}$ & $\begin{array}{r}169 \\
59,2 \\
78,1(8,9)\end{array}$ \\
\hline Zona geográfica (\%) & $\begin{array}{l}\text { Rural } \\
\text { Urbana }\end{array}$ & $\begin{array}{l}16,4 \\
83,6\end{array}$ & $\begin{array}{l}23,7 \\
76,3\end{array}$ \\
\hline Nivel educacional (\%) & $\begin{array}{l}\text { Básica } \\
\text { Media } \\
\text { Técnico-universitaria }\end{array}$ & $\begin{array}{r}59,7 \\
32,3 \\
8,0\end{array}$ & $\begin{array}{r}86,8 \\
12,0 \\
1,2\end{array}$ \\
\hline Nivel de ingreso (\%) & $\begin{array}{l}\text { Bajo } \\
\text { Medio } \\
\text { Alto }\end{array}$ & $\begin{array}{r}68,9 \\
24,9 \\
6,2\end{array}$ & $\begin{array}{r}84,3 \\
15,7 \\
0\end{array}$ \\
\hline Antropométricas & $\begin{array}{l}\text { Peso corporal (kg) } \\
\text { IMC }\left(\mathrm{kg} / \mathrm{m}^{2}\right)\end{array}$ & $\begin{array}{r}70,5(14,2) \\
28,7(5,4)\end{array}$ & $\begin{array}{r}65,8(14,6) \\
27,6(5,2)\end{array}$ \\
\hline Estado nutricional (\%) & $\begin{array}{l}\text { Bajo peso } \\
\text { Normal } \\
\text { Sobrepeso } \\
\text { Obesidad } \\
\text { Perímetro de cintura (cm) } \\
\text { Obesidad abdominal (\%) }\end{array}$ & $\begin{array}{r}10,6 \\
34,8 \\
25,5 \\
29,1 \\
99,8(12,0) \\
56,4\end{array}$ & $\begin{array}{r}21,2 \\
17,0 \\
27,3 \\
34,5 \\
98,3(12,8) \\
58,5\end{array}$ \\
\hline Estilo de vida & $\begin{array}{l}\text { Actividad física total (MET/hora/semana) } \\
\text { Actividad física de transporte (min/día) } \\
\text { Actividad física moderada (min/día) } \\
\text { Actividad física vigorosa (min/día) } \\
\text { Prevalencia inactividad física (\%) } \\
\text { Tiempo sedente (hora/día) } \\
\text { Consumo de frutas y vegetales (g/día) } \\
\text { Consumo de alcohol (g/día) } \\
\text { Promedio horas de sueño día }\end{array}$ & $\begin{array}{r}77,6(110,2) \\
36,4(66,3) \\
75,8(121,6) \\
27,0(84,1) \\
33,3 \\
3,3(2,5) \\
228,2(142,4) \\
40,4(70,0) \\
7,4(1,7)\end{array}$ & $\begin{array}{r}40,6(86,4) \\
23,7(64,6) \\
27,2(67,8) \\
18,1(70,6) \\
65,9 \\
5,0(3,2) \\
210,3(137,0) \\
39,7(41,8) \\
8,1(2,4)\end{array}$ \\
\hline Horas de sueño $(\%)$ & $\begin{array}{l}<7 \text { horas } \\
7-9 \text { horas } \\
>9 \text { horas }\end{array}$ & $\begin{array}{l}48,7 \\
31,6 \\
19,7\end{array}$ & $\begin{array}{l}30,8 \\
28,4 \\
40,8\end{array}$ \\
\hline Autorreporte salud y bienestar (\%) & $\begin{array}{l}\text { Malo } \\
\text { Regular } \\
\text { Bueno }\end{array}$ & $\begin{array}{r}2,8 \\
36,1 \\
61,1\end{array}$ & $\begin{array}{l}12,5 \\
50,0 \\
37,5\end{array}$ \\
\hline Tabaquismo (\%) & $\begin{array}{l}\text { Nunca } \\
\text { Exfumador } \\
\text { Fumador }\end{array}$ & $\begin{array}{l}51,6 \\
32,2 \\
16,2\end{array}$ & $\begin{array}{r}59,5 \\
31,6 \\
8,9\end{array}$ \\
\hline Metabólicas & $\begin{array}{l}\text { Síndrome metabólico (\%) } \\
\text { Diabetes mellitus tipo } 2(\%) \\
\text { Hipertensión arterial }(\%)\end{array}$ & $\begin{array}{l}51,5 \\
23,9 \\
68,5\end{array}$ & $\begin{array}{l}54,9 \\
33,1 \\
81,7\end{array}$ \\
\hline Problemas de audición & $\begin{array}{l}\text { No escucha normal por los dos oídos (\%) } \\
\text { No es capaz de seguir un programa de televisión a un volumen } \\
\text { aceptable }(\%) \\
\text { No es capaz de seguir una conversación con tres o más personas (\%) } \\
\text { Uno o más de un problema de audición (\%) } \\
\text { Tiene los tres problemas de audición estudiados (\%) }\end{array}$ & $\begin{array}{l}15,9 \\
46,0 \\
10,8\end{array}$ & $\begin{array}{l}54,2 \\
41,1 \\
42,3 \\
66,7 \\
26,2\end{array}$ \\
\hline
\end{tabular}


Tabla 2. Asociación entre MMSE alterado y distintos tipos de discapacidad auditiva

\begin{tabular}{|c|c|c|c|c|c|c|c|}
\hline Modelos & $\begin{array}{l}\text { No posee } \\
\text { discapacidad } \\
\text { auditiva }\end{array}$ & $\begin{array}{l}\text { No escucha } \\
\text { normal por } \\
\text { los dos oídos }\end{array}$ & Valor $p$ & $\begin{array}{l}\text { No es capaz de } \\
\text { seguir un programa } \\
\text { de televisión a un } \\
\text { volumen aceptable }\end{array}$ & Valor $p$ & $\begin{array}{l}\text { No es capaz de } \\
\text { seguir una } \\
\text { conversación } \\
\text { con tres o más } \\
\text { personas }\end{array}$ & Valor $p$ \\
\hline Modelo 0 & 1,00 (Ref.) & $1,70(1,22 ; 2,35)$ & 0,001 & $2,44(1,74 ; 3,42)$ & $<0,0001$ & $3,88(2,76 ; 5,48)$ & $<0,0001$ \\
\hline Modelo 1 & 1,00 (Ref.) & $1,33(0,94 ; 1,90)$ & 0,111 & $1,66(1,14 ; 2,41)$ & 0,008 & $2,62(1,80 ; 3,84)$ & $<0,0001$ \\
\hline Modelo 2 & 1,00 (Ref.) & $1,34(0,94 ; 1,90)$ & 0,109 & $1,66(1,14 ; 2,41)$ & 0,008 & $2,63(1,80 ; 3,84)$ & $<0,0001$ \\
\hline Modelo 3 & 1,00 (Ref.) & $1,39(0,95 ; 2,04)$ & 0,089 & $1,93(1,29 ; 2,88)$ & 0,001 & $2,83(1,88 ; 4,25)$ & $<0,0001$ \\
\hline Modelo 4 & 1,00 (Ref.) & $1,32(0,89 ; 1,95)$ & 0,169 & $1,76(1,16 ; 2,66)$ & 0,007 & $2,59(1,70 ; 3,95)$ & $<0,0001$ \\
\hline
\end{tabular}

Datos presentados como OR y sus respectivos intervalos de confianza ( $95 \%$ IC). Ref.: el grupo de referencia fue asignado a personas que no poseían discapacidades auditivas. Un valor mayor a 1 indica que hay un mayor riesgo de deterioro cognitivo (MMSE <13) en personas con distintos tipos de discapacidades auditivas. Los modelos fueron ajustados por: Modelo 0 - ajustado por sexo; Modelo 1 -ajustado por Modelo 0, pero también por nivel educacional; Modelo 2 - ajustado por el Modelo 1, pero también por tabaquismo; Modelo 3 - ajustado por el Modelo 2, pero también por IMC; Modelo 4 - ajustado por el Modelo 2, pero también por perímetro de cintura.

Tabla 3. Asociación entre MMSE alterado y acumulación de distintas discapacidades auditiva

\begin{tabular}{|c|c|c|c|c|c|c|c|c|c|}
\hline Modelos & $\begin{array}{c}\text { No posee } \\
\text { discapacidad } \\
\text { auditiva }\end{array}$ & $\begin{array}{c}\text { Tiene } 1 \\
\text { discapacidad }\end{array}$ & Valor $p$ & $\begin{array}{c}\text { Tiene } 2 \\
\text { discapacidades } \\
\text { auditivas }\end{array}$ & Valor $p$ & $\begin{array}{c}\text { Tiene } 3 \\
\text { discapacidades } \\
\text { auditivas }\end{array}$ & Valor $p$ & $\begin{array}{c}\mathrm{OR} \\
\text { tendencia }\end{array}$ & Valor $p$ \\
\hline Modelo 0 & 1,00 (Ref.) & $1,50(0,97 ; 2,33)$ & 0,069 & $2,61(1,62 ; 4,20)$ & $<0,0001$ & $3,91(2,53 ; 6,06)$ & $<0,0001$ & $1,59(1,38 ; 1,82)$ & $<0,0001$ \\
\hline Modelo 1 & 1,00 (Ref.) & $1,44(0,90 ; 2,29)$ & 0,127 & $1,84(1,09 ; 3,10)$ & 0,023 & $2,38(1,47 ; 3,86)$ & $<0,0001$ & $1,34(1,15 ; 1,56)$ & $<0,0001$ \\
\hline Modelo 2 & 1,00 (Ref.) & $1,44(0,90 ; 2,30)$ & 0,124 & $1,83(1,08 ; 3,09)$ & 0,024 & $2,38(1,47 ; 3,86)$ & $<0,0001$ & $1,34(1,15 ; 1,56)$ & $<0,0001$ \\
\hline Modelo 3 & 1,00 (Ref.) & $1,33(0,79 ; 2,24)$ & 0,278 & $2,08(1,19 ; 3,62)$ & 0,010 & $2,63(1,57 ; 4,41)$ & $<0,0001$ & $1,39(1,18 ; 1,64)$ & $<0,0001$ \\
\hline Modelo 4 & 1,00 (Ref.) & $1,17(0,68 ; 2,00)$ & 0,579 & $1,75(0,99 ; 3,09)$ & 0,053 & $2,38(1,40 ; 4,02)$ & 0,001 & $1,34(1,13 ; 1,58)$ & 0,001 \\
\hline
\end{tabular}

Datos presentados como OR y sus respectivos intervalos de confianza ( $95 \%$ IC). Ref.: el grupo de referencia fue asignado a personas que no poseían discapacidades auditivas. Un valor mayor a 1 indica que hay un mayor riesgo de deterioro cognitivo (MMSE <13) en personas con una 0 más de una discapacidad auditiva. El OR para tendencia indica cuál es la probabilidad de presentar deterioro cognitivo en la medida que aumentan las discapacidades auditivas. Los modelos fueron ajustados por: Modelo 0 - ajustado por sexo; Modelo 1 -ajustado por Modelo 0, pero también por nivel educacional; Modelo 2 - ajustado por el Modelo 1, pero también por tabaquismo; Modelo 3 - ajustado por el Modelo 2, pero también por IMC; Modelo 4 - ajustado por el Modelo 2, pero también por perímetro de cintura.

yores ${ }^{13,22-24}$, éste es el primer estudio que identifica la fuerte asociación entre estas dos condiciones. Sin embargo, similares resultados han sido reportados en poblaciones norteamericanas, europeas y asiáticas ${ }^{7,8,25}$. Yuan y cols., identificaron que adultos mayores con discapacidad auditiva periférica y central poseían un mayor riesgo de deterioro cognitivo en comparación con aquellos con función auditiva normal ${ }^{7}$. También demostraron una tendencia al deterioro cognitivo en la medida que aumentan los umbrales de audición? ${ }^{7}$. Davidson y Guthrie evidenciaron que adultos mayores que además de presentar problemas auditivos presentaban discapacidad visual, poseían una mayor probabilidad de desarrollar demencia y dificultades comunicativas ${ }^{8}$. Resultados similares fueron identificados en 45.805 adultos mayores británicos, en quienes tanto la discapacidad auditiva como visual fue un marcador de riesgo de deterioro cognitivo ${ }^{25}$.

Considerando que la pérdida de la audición podría acelerar el declive cognitivo relacionado con la edad, abordar en forma más exhaustiva la pérdida de audición podría ayudar a retrasar el deterioro cognitivo y la demencia6,21. Se ha propuesto que intervenciones de rehabilitación auditiva integral, que incorporen el uso de ayudas sensoriales para maximizar la audibilidad de las señales de voz, podrían reducir de forma plausible la carga cogniti- 
$\mathrm{va}^{26}$. Para determinar los efectos de un tratamiento auditivo sobre la disminución de la capacidad cognitiva, Castiglione y cols. estudiaron los efectos de la rehabilitación mediante implantes cocleares 0 audífonos en 125 adultos mayores con diferente grado de pérdida de audición ${ }^{27}$. Luego de la rehabilitación, los grupos presentaron una mejoría en las tareas de memoria a corto y largo plazo, en el nivel de depresión y puntajes de estado cognitivo, evidenciando los positivos efectos cognitivos al proporcionar una audición efectiva ${ }^{27}$.

En Chile, como parte de las garantías explicitas de salud, existe un tratamiento garantizado para las personas de 65 años y más que presenten hipoacusia bilateral y que requieran el uso de audífono ${ }^{20}$, lo que podría ser una excelente solución para disminuir las complicaciones asociadas, ya que, como se ha demostrado, hipoacusias mayores a 40 decibeles son un factor de riesgo importante para el desarrollo de demencia ${ }^{28,29}$. No obstante, aún hay muchos desafíos y aspectos en los que se debe continuar avanzado en relación con esta patología. Entre ellos, la entrega y adherencia al uso de audífonos por parte de los adultos mayores de 65 años (se ha estimado solo $53 \%$ de adherencia en el sistema público ${ }^{30}$ ), así como también mejorar el screening diagnóstico de esta patología, ya que se estima que solamente el $11,8 \%$ de los médicos de atención primaria realizan este tamizaje anualmente y que solo el $17,6 \%$ lo realizan ante una sospecha de un problema auditivo $0^{23,31}$.

Entre las fortalezas de este estudio se encuentran el uso del MMSE abreviado, el cual ha sido validado anteriormente en población chilena ${ }^{13}$, así como también la medición de la percepción auditiva a través de preguntas previamente validadas en la ENS $2003^{32}$. Sin embargo, entre las limitacio-

\section{BIBLIOGRAFÍA}

1. Pinto JM, Kern DW, Wroblewski Ke, Chen RC, Schumm LP, McCLIntock MK. Sensory function: insights from Wave 2 of the National Social Life, Health, and Aging Project. J Gerontol B Psychol Sci Soc Sci 2014; 69: S144-53. doi: 10.1093/ geronb/gbu102.

2. WHO. Sordera y pérdida de la audición. World Health Organization. 2018; Disponible: http:// nes es importante considerar que la discapacidad auditiva fue evaluada a través del autorreporte de preguntas y no a través de audiometría, similar al estudio de Amieva y cols $^{33}$. Este tipo de metodología podría haber generado que adultos mayores con problemas auditivos podrían no haber identificado y/o escuchado claramente las preguntas del test MMSE, ocasionando respuestas erróneas y, en consecuencia, un menor puntaje en el test. Otra limitación es la falta de diagnóstico de hipoacusia, lo cual hubiese confirmado la presencia de discapacidad auditiva por parte de los individuos y no solamente alteración en la percepción auditiva. Por otro lado, y en relación con el MMSE abreviado, un reciente estudio desarrollado por Jiménez y cols. ha evidenciado que este instrumento presenta una baja sensibilidad y un bajo poder discriminativo, demostrando que podría ser insuficiente para ser utilizado como método de detección de demencia en adultos mayores ${ }^{34}$. Adicionalmente, debido al diseño observacional de corte transversal de la ENS 2009-2010, no se puede establecer una relación de causa-efecto entre las variables.

\section{CONCLUSIÓN}

La disminución de la percepción auditiva es un factor de riesgo para el desarrollo de deterioro cognitivo y posteriormente demencia. La creación de políticas públicas, orientadas al tamizaje temprano en población de riesgo, podría ser una solución efectiva para prevenir las consecuencias asociadas con esta condición, sobre todo considerando que estas patologías influyen directamente en el bienestar emocional y social de los adultos mayores.

www.who.int/es/news-room/fact-sheets/detail/ deafness-and-hearing-loss.

3. WHO. Demencia. World Health Organization. 2017; Disponible: http://www.who.int/es/newsroom/fact-sheets/detail/dementia.

4. Crews Je, Campbell VA. Vision impairment and hearing loss among community-dwelling older Americans: implications for health and functioning. Am J Public Health 2004; 94: 823-9.

5. Fulton SE, Lister JJ, Bush AL, Edwards JD, 
Andel R. Mechanisms of the Hearing-Cognition Relationship. Semin Hear 2015; 36: 140-9.

6. Bernabel R, Bonuccelli U, Maggi S, Marengoni A, Martini A, Memo M, et al. Hearing loss and cognitive decline in older adults: questions and answers. Aging Clin Exp Res 2014; 26: 567-73.

7. Yuan J, Sun Y, Sang S, Pham JH, Kong WJ. The risk of cognitive impairment associated with hearing function in older adults: a pooled analysis of data from eleven studies. Sci Rep 2018; 8: 2137.

8. Davidson JGS, Guthrie DM. Older Adults With a Combination of Vision and Hearing Impairment Experience Higher Rates of Cognitive Impairment, Functional Dependence, and Worse Outcomes Across a Set of Quality Indicators. J Aging Health 2017; 898264317723407.

9. Kiely KM, Gopinath B, Mitchell P, Luszcz M, Anstey KJ. Cognitive, health, and sociodemographic predictors of longitudinal decline in hearing acuity among older adults. J Gerontol A Biol Sci Med Sci 2012; 67: 997-1003.

10. Woodford HJ, George J. Cognitive assessment in the elderly: a review of clinical methods. QJM 2007; 100: 469-84.

11. Folstein MF, Folstein SE, McHugh PR. "Minimental state". A practical method for grading the cognitive state of patients for the clinician. J Psychiatr Res 1975; 12: 189-98.

12. ICAZA MG, CA. Minimental State Examination: Análisis estadístico del estudio de demencia en Chile para validar una versión abreviada. Investigaciones en Salud Publica: Documento Técnico. OPS, Washington, DC. 1999; Disponible: http://www.bvsde.paho.org/texcom/ cd045364/049147.pdf.

13. Quiroga L P, Albala B C, Klaasen P G. Validación de un test de tamizaje para el diagnóstico de demencia asociada a edad, en Chile. Rev Med Chil 2004; 132: 467-78.

14. MINSAL. Orientación técnica para la atención de salud de las personas adultas mayores en atención primaria. Ministerio de Salud, Chile. 2014; Disponible: http://www.minsal.cl/sites/ default/files/files/ot_a_MAYOR.pdf.

15. MINSAL. Chile: Encuesta Nacional de Salud 2009-2010. Ministerio de Salud de Chile. 2010; Disponible en http://web.minsal.cl/portal/url/ item/bcb03d7bc28b64dfe040010165012d23. pdf.
16. ADA. Report of the Expert Committee on the Diagnosis and Classification of Diabetes Mellitus. Diabetes Care 1997; 20: 1183-97.

17. Grundy SM, Cleeman JI, Merz CN, Brewer HB, JR., Clark LT, Hunninghake DB, et al. Implications of recent clinical trials for the National Cholesterol Education Program Adult Treatment Panel III guidelines. Circulation 2004; 110: 227-39.

18. OPS. Parte 1: Módulos de Valoración clínica. Módulo 5: Valoración Nutricional del Adulto Mayor. Organización Panamericana de la Salud. 2003.

19. WHO. Global Physical Activity Questionnaire: GPAQ version 2.0. World Health Organization. 2009; Disponible en: http://www.who.int/chp/ steps/resources/GPAQ_Analysis_Guide.pdf.

20. MINSAL. Guía Clinica Auge: Hipoacusia bilateral en personas de 65 años y más que requieren uso de audífono. Ministerio de Salud, Chile. 2013; Disponible en : http://www.bibliotecaminsal.cl/ wp/wp-content/uploads/2016/04/Hipoacusiabilateral-mayores-65-a\%C3\%B10s.pdf.

21. Livingston G, Sommerlad A, Orgeta V, Costafreda SG, Huntley J, Ames D, et Al. Dementia prevention, intervention, and care. Lancet 2017; 390: 2673734.

22. Cardemil F, Barría T, Aguayo L, Esquivel P, Rahal M, Fuente A, et Al. Evaluación del programa "Active Communication Education" para rehabilitación auditiva en adultos mayores con hipoacusia usuarios de audífonos. Rev Otorrinolaringol Cir Cabeza Cuello 2014; 74: 93-100.

23. CardemiL MF. Hipoacusia asociada al envejecimiento en Chile: ¿En qué aspectos se podría avanzar? Rev Otorrinolaringol Cir Cabeza Cuello 2016; 76: 127-35.

24. Donoso A, Venegas P, Villarroel $C$, Vásquez $C$. Deterioro cognitivo leve y enfermedad de Alzheimer inicial en adultos mayores. Rev Chil Neuro-Psiquiatr 2001; 39:231-8.

25. Maharani A, Dawes P, Nazroo J, Tampubolon G, Pendeteton N. Visual and hearing impairments are associated with cognitive decline in older people. Age Ageing 2018, en prensa. doi: 10.1093/ ageing/afy061.

26. Sarampalis A, Kalluri S, Edwards B, Hafter E. Objective measures of listening effort: effects of background noise and noise reduction. J Speech Lang Hear Res 2009; 52: 1230-40. 
27. Castiglione A, Benatti A, Velardita C, Favaro D, Padoan E, Severi D, et al. Aging, Cognitive Decline and Hearing Loss: Effects of Auditory Rehabilitation and Training with Hearing Aids and Cochlear Implants on Cognitive Function and Depression among Older Adults. Audiol Neurootol 2016; 21: 21-8.

28. Délano P. Hipoacusia: Un nuevo factor de riesgo para demencia. Rev Otorrinolaringol Cir Cabeza Cuello 2017; 77: 237-8.

29. Deal Ja, Betz J, Yaffe K, Harris T, PurchaseHelzner E, Satterfield S, et al. Hearing Impairment and Incident Dementia and Cognitive Decline in Older Adults: The Health ABC Study. J Gerontol A Biol Sci Med Sci 2017; 72: 703-9.

30. MINSAL. Encuesta Nacional de Salud 2003. Ministerio de Salud, Gobierno de Chile. 2003.
31. Cohen SM, Labadie RF, Haynes DS. Primary care approach to hearing loss: the hidden disability. Ear Nose Throat J 2005; 84: 26, 9-31, 44.

32. MINSAL. Encuesta Nacional de Salud 2003. Ministerio de Salud, Chile. 2003; Disponible en: http://www.medicinadefamiliares.cl/Protocolos/ encnacsalres.pdf.

33. Amieva H, Ouvrard C, Meillon C, Rullier L, DARTIGUES JF. Death, Depression, Disability and Dementia Associated with Self-Reported Hearing Problems: a 25-Year Study. J Gerontol A Biol Sci Med Sci 2018; 73: 1383-9.

34. Jimenez D, Lavados M, Rojas P, Henriquez C, Silva F, Guilzon M. [Performance of an abbreviated mini mental examination to detect dementia in older people]. Rev Med Chil 2017; 145: 862-8.

Correspondencia: Carlos Celis-Morales Glasgow Cardiovascular Research Centre Institute of Cardiovascular and Medical Science University of Glasgow

G12 8TA, Glasgow, Reino Unido

E mail: Carlos.Celis@glasgow.ac.uk 